International Journal of Pure and Applied Mathematics

Volume 90 No. 4 2014, 413-421

ISSN: 1311-8080 (printed version); ISSN: 1314-3395 (on-line version)

url: http://www.ijpam.eu

doi: http://dx.doi.org/10.12732/ijpam.v90i4.3

\title{
DETERMINANT OF ADJACENCY MATRIX OF SQUARE CYCLE GRAPH
}

\author{
Nitiphoom Adsawatithisakul ${ }^{1}$, Decha Samana ${ }^{2} \S$ \\ ${ }^{1,2}$ Department of Mathematics \\ Faculty of Science \\ King Mongkut's Institute of Technology Ladkrabang \\ Bangkok 10520, THAILAND
}

Abstract: Square Cycle, $C_{n}^{2}$ is a graph that has $n$ vertices and two vertices $u$ and $v$ are adjacent if and only if distance between $u$ and $v$ not greater than 2. In this paper, we show that the determinant of adjacency matrix of square cycle $C_{n}^{2}$ are as follows

$$
\operatorname{det}\left(A\left(C_{n}^{2}\right)\right)= \begin{cases}0, & n \equiv 0,2,4 \bmod 6 \\ 16, & n \equiv 3 \bmod 6 \\ 4, & n \equiv 1,5 \bmod 6 .\end{cases}
$$

AMS Subject Classification: $05 \mathrm{C} 50$

Key Words: determinant, square cycle graph, adjacency matrix

\section{Introduction}

Let $G$ be a simple graph with $n$ vertices. We denote $\operatorname{det}(A(G))$ is the determinant of adjacency matrix of $G$ and $E(G ; k)$ is $k^{t h}$ eigenvalues of the adjacency matrix which $\operatorname{det}(A(G))$ and $E(G ; k)$ are independent of the choice of vertices

Received: May 21, 2013

(c) 2014 Academic Publications, Ltd.

$\S$ Correspondence author url: www.acadpubl.eu 



Figure 1: $d$-th power of cycle graph

in adjacency matrix and are an invariant of $G$.

In [2] and [4], they determined the determinant of adjacency matrix of some graphs, such as $K_{n}, C_{n}, P_{n}$ and $W_{n}$. B. Gyurov and J. Cloud [7] has determined determinant of Pin-wheel graph. Moreover, there are studies of graph which satisfy some properties of determinant for example, M. Doob [5] construct circulant graph with $\operatorname{det}(A(G))=-\operatorname{deg}(G)$, S. Hu [9] and A. Abdollahi [1] have found that the determinant of graphs with exactly one cycle and exactly two cycles, respectively.

Cycle power, $C_{n}^{d}$ is a graph that has $n$ vertices and distance each pair of vertex is less or equal $d$. For example,

If $d=2, n \geq 6$, it is called square cycle graph.

Furthermore, there are studies of cycle power such as: C.N. Campos and C.P. de Mello [3], M. Krivelevich and A. Nachmias [10] studied about the colouring in cycle power, Y. Hoa, C. Woo and P. Chen [8] investigate the sandpile group in cycle power, D. Li and M. Liu [11] consider cycle power and their complements which satisfy Hadwiger's conjecture.

From figure 1 graph $C_{6}^{2}$ and graph $C_{8}^{2}$, we write to adjacency matrix

$$
A\left(C_{6}^{2}\right)=\left[\begin{array}{llllll}
0 & 1 & 1 & 0 & 1 & 1 \\
1 & 0 & 1 & 1 & 0 & 1 \\
1 & 1 & 0 & 1 & 1 & 0 \\
0 & 1 & 1 & 0 & 1 & 1 \\
1 & 0 & 1 & 1 & 0 & 1 \\
1 & 1 & 0 & 1 & 1 & 0
\end{array}\right], \quad A\left(C_{8}^{2}\right)=\left[\begin{array}{llllllll}
0 & 1 & 1 & 0 & 0 & 0 & 1 & 1 \\
1 & 0 & 1 & 1 & 0 & 0 & 0 & 1 \\
1 & 1 & 0 & 1 & 1 & 0 & 0 & 0 \\
0 & 1 & 1 & 0 & 1 & 1 & 0 & 0 \\
0 & 0 & 1 & 1 & 0 & 1 & 1 & 0 \\
0 & 0 & 0 & 1 & 1 & 0 & 1 & 1 \\
1 & 0 & 0 & 0 & 1 & 1 & 0 & 1 \\
1 & 1 & 0 & 0 & 0 & 1 & 1 & 0
\end{array}\right] .
$$

We see adjacency matrix of $C_{6}^{2}$ and $C_{8}^{2}$ is a circulant matrix because a main diagonal of matrix is equal to zero and entries in first row satisfy $a_{1 j}=a_{1,(n-j+2)}$ 
for $j=2, \ldots, n$ and $a_{i j}=a_{i+1, j+1}$, then a square cycle graph is a circulant graph. It is interesting to study determinant of adjacency matrix of square cycle graph.

Proposition 1. (see [2]) Suppose that $\left[0, a_{2}, \ldots, a_{n}\right]$ is the first row of the adjacency matrix of a circulant graph $G$. Then the eigenvalues of graph $G$ is denoted $E(G ; k)$,

$$
E(G ; k)=\sum_{j=1}^{n} a_{j} z^{j-1}
$$

where $z=e^{\frac{2 k \pi i}{n}}, k=1,2, \ldots, n$.

Square cycle graph is a circulant graph then eigenvalues of square cycle graph is

$$
E\left(C_{n}^{2} ; k\right)=z+z^{2}+z^{n-2}+z^{n-1} .
$$

Determinant of a square matrix can be find by eigenvalue its as below

Theorem 2. (see [6]) Let $\lambda_{1}, \ldots, \lambda_{n}$ be a eigenvalues of a square matrix $A$. Then

$$
\operatorname{det}(A)=\lambda_{1} \lambda_{2} \ldots \lambda_{n} .
$$

Next, we present lemma that will be used in the proof of determinant of adjacency matrix of square cycle graph.

\section{Main Results}

Lemma 3. Let $q$ be a positive number. Then

$$
\begin{aligned}
& \prod_{k=1}^{2 q}\left(\cos \frac{3 k \pi}{6 q+3} \cos \frac{k \pi}{6 q+3}\right) \prod_{k=2 q+2}^{4 q+1}\left(\cos \frac{3 k \pi}{6 q+3} \cos \frac{k \pi}{6 q+3}\right) \\
& \prod_{k=4 q+3}^{6 q+2}\left(\cos \frac{3 k \pi}{6 q+3} \cos \frac{k \pi}{6 q+3}\right)=2^{-12 q} .
\end{aligned}
$$

Proof. The left hand side of (2) is

$$
\prod_{k=1}^{2 q} \frac{\sin \frac{6 k \pi}{6 q+3} \sin \frac{2 k \pi}{6 q+3}}{2 \sin \frac{3 k \pi}{6 q+3} 2 \sin \frac{k \pi}{6 q+3}} \prod_{k=2 q+2}^{4 q+1} \frac{\sin \frac{6 k \pi}{6 q+3} \sin \frac{2 k \pi}{6 q+3}}{2 \sin \frac{3 k \pi}{6 q+3} 2 \sin \frac{k \pi}{6 q+3}} \prod_{k=4 q+3}^{6 q+2} \frac{\sin \frac{6 k \pi}{6 q+3} \sin \frac{2 k \pi}{6 q+3}}{2 \sin \frac{3 k \pi}{6 q+3} 2 \sin \frac{k \pi}{6 q+3}}
$$




$$
\begin{aligned}
&=\frac{1}{2^{12 q}}(\left.\frac{\prod_{k=q+1}^{2 q} \sin \frac{6 k \pi}{6 q+3} \sin \frac{2 k \pi}{6 q+3}}{\prod_{k=1}^{q} \sin \frac{(6 k-3) \pi}{6 q+3} \sin \frac{(2 k-1) \pi}{6 q+3}}\right) \\
&\left(\frac{\prod_{k=2 q+2}^{4 q+1} \sin \frac{6 k \pi}{6 q+3} \sin \frac{2 k \pi}{6 q+3}}{\prod_{k=2 q+2}^{4 q+1} \sin \frac{3 k \pi}{6 q+3} \sin \frac{k \pi}{6 q+3}}\right) \\
&\left(\frac{\prod_{k=4 q+3}^{6 q+2} \sin \frac{6 k \pi}{6 q+3} \sin \frac{2 k \pi}{6 q+3}}{\prod_{k=4 q+3}^{6 q+2} \sin \frac{3 k \pi}{6 q+3} \sin \frac{k \pi}{6 q+3}}\right) \\
&=\frac{1}{2^{12 q}}\left(\frac{\prod_{k=1}^{q} \sin \frac{(6 k-3) \pi}{6 q+3} \sin \frac{(2 k-1) \pi}{6 q+3}}{\prod_{k=1}^{q} \sin \frac{(6 k-3) \pi}{6 q+3} \sin \frac{(2 k-1) \pi}{6 q+3}}\right) \\
& \\
&\left(\frac{\prod_{k=2 q+2}^{3 q+1} \sin \frac{(6 k-(6 q+3)) \pi}{6 q+3} \sin \frac{(2 k-(2 q+1)) \pi}{6 q+3}}{\prod_{k=2 q+2}^{3 q+1} \sin \frac{(6 k-(6 q+3)) \pi}{6 q+3} \sin \frac{(2 k-(2 q+1)) \pi}{6 q+3}}\right) \\
&\left(\frac{\prod_{k=4 q+3}^{5 q+2} \sin \frac{(6 k-(12 q+9)) \pi}{6 q+3} \sin \frac{(2 k-(4 q+3)) \pi}{6 q+3}}{\prod_{k=4 q+3}^{5 q+2} \sin \frac{(6 k-(12 q+9)) \pi}{6 q+3} \sin \frac{(2 k-(4 q+3)) \pi}{6 q+3}}\right) \\
&=2^{-12 q .}
\end{aligned}
$$

Lemma 4. Let $q$ be a positive integer. Then

$$
\prod_{k=1}^{6 q}\left(\cos \frac{3 k \pi}{6 q+1} \cos \frac{k \pi}{6 q+1}\right)=2^{-12 q} .
$$

Proof. It can be proved by

$$
\begin{aligned}
\prod_{k=1}^{6 q}\left(\cos \frac{3 k \pi}{6 q+1} \cos \frac{k \pi}{6 q+1}\right) & =\prod_{k=1}^{6 q} \frac{\sin \frac{6 k \pi}{6 q+1} \sin \frac{2 k \pi}{6 q+1}}{2 \sin \frac{3 k \pi}{6 q+1} 2 \sin \frac{k \pi}{6 q+1}} \\
& =\frac{1}{2^{12 q}}\left(\frac{\prod_{k=3 q+1}^{6 q} \sin \frac{6 k \pi}{6 q+1} \sin \frac{2 k \pi}{6 q+1}}{\prod_{k=1}^{3 q} \sin \frac{(6 k-3) \pi}{6 q+1} \sin \frac{(2 k-1) \pi}{6 q+1}}\right) \\
& =\frac{1}{2^{12 q}}\left(\frac{\prod_{k=1}^{3 q} \sin \frac{(6 k-3) \pi}{6 q+1} \sin \frac{(2 k-1) \pi}{6 q+1}}{\prod_{k=1}^{3 q} \sin \frac{(6 k-3) \pi}{6 q+1} \sin \frac{(2 k-1) \pi}{6 q+1}}\right) \\
& =2^{-12 q} .
\end{aligned}
$$

Lemma 5. Let $q$ be a positive integer. Then 


$$
\prod_{k=1}^{6 q+4}\left(\cos \frac{3 k \pi}{6 q+5} \cos \frac{k \pi}{6 q+5}\right)=2^{-2(6 q+4)}
$$

Proof. It can be proved by

$$
\begin{aligned}
\prod_{k=1}^{6 q+4}\left(\cos \frac{3 k \pi}{6 q+5} \cos \frac{k \pi}{6 q+5}\right) & =\prod_{k=1}^{6 q+4} \frac{\sin \frac{6 k \pi}{6 q+5} \sin \frac{2 k \pi}{6 q+5}}{2 \sin \frac{3 k \pi}{6 q+5} 2 \sin \frac{k \pi}{6 q+5}} \\
& =\frac{1}{2^{2(6 q+4)}}\left(\frac{\prod_{k=3 q+3}^{6 q+4} \sin \frac{6 k \pi}{6 q+5} \sin \frac{2 k \pi}{6 q+5}}{\prod_{k=1}^{3 q+2} \sin \frac{(6 k-3) \pi}{6 q+5} \sin \frac{(2 k-1) \pi}{6 q+5}}\right) \\
& =\frac{1}{2^{2(6 q+4)}}\left(\frac{\prod_{k=1}^{3 q+2} \sin \frac{(6 k-3) \pi}{6 q+5} \sin \frac{(2 k-1) \pi}{6 q+5}}{\prod_{k=1}^{3 q+2} \sin \frac{(6 k-3) \pi}{6 q+5} \sin \frac{(2 k-1) \pi}{6 q+5}}\right) \\
& =2^{-2(6 q+4)}
\end{aligned}
$$

Next, we use Lemma 3, 4 and 5 to find determinant of adjacency matrix of square cycle graph.

Theorem 6. Let $C_{n}^{2}$ be a square cycle graph with $n$ vertices and $n$ be a positive integer. Then

$$
\operatorname{det}\left(A\left(C_{n}^{2}\right)\right)= \begin{cases}0, & n \equiv 0,2,4 \bmod 6 \\ 16, & n \equiv 3 \bmod 6 \\ 4, & n \equiv 1,5 \bmod 6\end{cases}
$$

Proof. Let $E\left(C_{n}^{2} ; k\right)$ be a $k^{\text {th }}$ eigenvalue of adjacency matrix of square cycle graph $C_{n}^{2}$. From (1), We get

$$
\begin{aligned}
E\left(C_{n}^{2} ; k\right) & =e^{\frac{2 k \pi i}{n}}+e^{\frac{4 k \pi i}{n}}+e^{\frac{2 k(n-2) \pi i}{n}}+e^{\frac{2 k(n-1) \pi i}{n}} \\
& =e^{\frac{2 k \pi i}{n}}+e^{\frac{4 k \pi i}{n}}+e^{\frac{2 k n \pi i}{n}} \cdot e^{\frac{-4 k \pi i}{n}}+e^{\frac{2 k n \pi i}{n}} \cdot e^{\frac{-2 k \pi i}{n}} .
\end{aligned}
$$

By Euler's formula, we obtain

$$
\begin{aligned}
E\left(C_{n}^{2} ; k\right) & =\left(\cos \frac{2 k \pi}{n}+i \sin \frac{2 k \pi}{n}\right)+\left(\cos \frac{4 k \pi}{n}+i \sin \frac{4 k \pi}{n}\right)+ \\
& \left(\cos \frac{-4 k \pi}{n}+i \sin \frac{-4 k \pi}{n}\right)+\left(\cos \frac{-2 k \pi}{n}+i \sin \frac{-2 k \pi}{n}\right) \\
& =2 \cos \frac{2 k \pi}{n}+2 \cos \frac{4 k \pi}{n}
\end{aligned}
$$


We can rewrite

$$
E\left(C_{n}^{2} ; k\right)=4\left(\cos \frac{3 k \pi}{n} \cos \frac{k \pi}{n}\right) .
$$

From (3) We have

$$
\begin{aligned}
\operatorname{det}\left(A\left(C_{n}^{2}\right)\right) & =\prod_{k=1}^{n} E\left(C_{n}^{2} ; k\right) \\
& =\prod_{k=1}^{n} 4\left(\cos \frac{3 k \pi}{n} \cos \frac{k \pi}{n}\right) .
\end{aligned}
$$

Consider $n$ as follows

Case I. $n \equiv 0,2,4 \bmod 6$.

Since $n$ is even and $1 \leq k \leq n$, consider (3) when $k=\frac{n}{2}$. Then

$$
\begin{aligned}
E\left(C_{n}^{2} ; \frac{n}{2}\right) & =4\left(\cos \frac{3 \frac{n}{2} \pi}{n} \cos \frac{\frac{n}{2} \pi}{n}\right) \\
& =0 .
\end{aligned}
$$

From (4), we obtain

$$
\begin{aligned}
\operatorname{det}\left(A\left(C_{n}^{2}\right)\right) & =\prod_{k=1}^{n} E\left(C_{n}^{2} ; k\right) \\
& =0 .
\end{aligned}
$$

Therefore, $\operatorname{det}\left(A\left(C_{n}^{2}\right)\right)=0$ when $n \equiv 0,2,4 \bmod 6$.

Case II. $n \equiv 3 \bmod 6$ Then $n=6 q+3, \exists q \in \mathbb{Z}^{+}$.

From (4), we obtain

$$
\begin{aligned}
\operatorname{det}\left(A\left(C_{n}^{2}\right)\right)= & \prod_{k=1}^{n} E\left(C_{n}^{2} ; k\right) \\
= & \prod_{k=1}^{6 q+3} 4\left(\cos \frac{3 k \pi}{n} \cos \frac{k \pi}{n}\right) \\
= & 4\left(\cos \frac{3(2 q+1) \pi}{6 q+3} \cos \frac{(2 q+1) \pi}{6 q+3}\right) 4\left(\cos \frac{3(4 q+2) \pi}{6 q+3} \cos \frac{(4 q+2) \pi}{6 q+3}\right) \\
& 4\left(\cos \frac{3(6 q+3) \pi}{6 q+3} \cos \frac{(6 q+3) \pi}{6 q+3}\right) 2^{12 q} \prod_{k=1}^{2 q} 4\left(\cos \frac{3 k \pi}{6 q+3} \cos \frac{k \pi}{6 q+3}\right)
\end{aligned}
$$




$$
\begin{aligned}
& \prod_{k=2 q+2}^{4 q+1} 4\left(\cos \frac{3 k \pi}{6 q+3} \cos \frac{k \pi}{6 q+3}\right) \prod_{k=4 q+3}^{6 q+2} 4\left(\cos \frac{3 k \pi}{6 q+3} \cos \frac{k \pi}{6 q+3}\right) \\
= & (-2)(-2)(4) 2^{12 q} \prod_{k=1}^{2 q} 4\left(\cos \frac{3 k \pi}{6 q+3} \cos \frac{k \pi}{6 q+3}\right) \\
& \prod_{k=2 q+2}^{4 q+1} 4\left(\cos \frac{3 k \pi}{6 q+3} \cos \frac{k \pi}{6 q+3}\right) \\
& \prod_{k=4 q+3}^{6 q+2} 4\left(\cos \frac{3 k \pi}{6 q+3} \cos \frac{k \pi}{6 q+3}\right) .
\end{aligned}
$$

Using Lemma 3, we have

$$
\begin{aligned}
\operatorname{det}\left(A\left(C_{n}^{2}\right)\right) & =(-2)(-2)(4)\left(2^{12 q}\right)\left(2^{-12 q}\right) \\
& =16 .
\end{aligned}
$$

Therefore $\operatorname{det}\left(A\left(C_{n}^{2}\right)\right)=16$ when $n \equiv 3 \bmod 6$.

Case III. $n \equiv 1 \bmod 6$ and $n \equiv 5 \bmod 6$. We consider 2 subcases.

Subcase $3.1, n \equiv 1 \bmod 6$, by (4), we obtain

$$
\begin{aligned}
\operatorname{det}\left(A\left(C_{n}^{2}\right)\right) & =\prod_{k=1}^{n} E\left(C_{n}^{2} ; k\right) \\
& =\prod_{k=1}^{6 q+1} 2^{2}\left(\cos \frac{3 k \pi}{6 q+1} \cos \frac{k \pi}{6 q+1}\right) \\
& =4\left(\cos \frac{3(6 q+1) \pi}{6 q+1} \cos \frac{(6 q+1) \pi}{6 q+1}\right) 2^{12 q}\left(\prod_{k=1}^{6 q} 4\left(\cos \frac{3 k \pi}{6 q+1} \cos \frac{k \pi}{6 q+1}\right) .\right.
\end{aligned}
$$

Using Lemma 4, we have

$$
\begin{aligned}
\operatorname{det}\left(A\left(C_{n}^{2}\right)\right) & =4\left(2^{12 q}\right)\left(2^{-12 q}\right) \\
& =4 .
\end{aligned}
$$

Subcase $3.2, n \equiv 5 \bmod 6$, by (4), we obtain

$$
\begin{aligned}
\operatorname{det}\left(A\left(C_{n}^{2}\right)\right) & =\prod_{k=1}^{n} E\left(C_{n}^{2} ; k\right) \\
& =\prod_{k=1}^{6 q+5} 2^{2}\left(\cos \frac{3 k \pi}{6 q+5} \cos \frac{k \pi}{6 q+5}\right)
\end{aligned}
$$




$$
=4\left(\cos \frac{3(6 q+5) \pi}{6 q+5} \cos \frac{(6 q+5) \pi}{6 q+5}\right) 2^{2(6 q+4)}\left(\prod_{k=1}^{6 q+4} 4\left(\cos \frac{3 k \pi}{6 q+5} \cos \frac{k \pi}{6 q+5}\right)\right) .
$$

Using Lemma 5, we have

$$
\begin{aligned}
\operatorname{det}\left(A\left(C_{n}^{2}\right)\right) & =4\left(2^{2(6 q+4)}\right)\left(2^{-2(6 q+4)}\right) \\
& =4 .
\end{aligned}
$$

From subcase 3.1 and 3.2 , we obtain

$$
\operatorname{det}\left(A\left(C_{n}^{2}\right)\right)=4 \text { for } n \equiv 1,5 \bmod 6 .
$$

From case I, II and III,

$$
\operatorname{det}\left(A\left(C_{n}^{2}\right)\right)= \begin{cases}0, & n \equiv 0,2,4 \bmod 6 \\ 16, & n \equiv 3 \bmod 6 \\ 4, & n \equiv 1,5 \bmod 6 .\end{cases}
$$

\section{References}

[1] A. Abdollahi, Determinant of adjacency matrices of graph, http://arxiv.org/abs/0908.3324, Cornell University (2009).

[2] N. Biggs, Algebraic Graph Theory, Cambridge University Press, Cambridge (1974).

[3] C.N. Campos and C.P. De Mello, A result on the total colouring of powers of cycles, Electronic Notes in Discrete Mathematics, 18 (2004), 47-52.

[4] D.M. Cvetkovic,M. Doob and H. Sachs, Spectra of Graphs: Theory and Application, Academic Press, New York, USA (1980).

[5] M. Doob, Circulant graphs with $\operatorname{det}(-A(G))=-\operatorname{deg}(G)$ :codeterminants with $K_{n}$, Linear Algebra and its Applications, 340 (2002), 87-96.

[6] L. Goldberg, Matrix Theory with Applications. McGraw - Hill International Editions, Mathematics and Statistics Series (1991).

[7] B. Gyurov and J. Cloud, On the algebraic properties of Pin-Wheel graphs and Applications, $73^{\text {rd }}$ annual meeting of the Oklahoma Arkansas section 2011, University of central Oklahoma, USA (2011).

[8] Y. Hoa, C. Woo and P.Chen, On the sandpile group of the square cycle $C_{n}^{2}$, Linear Algebra and its Applications, 418 (2006), 457-467. 
[9] S. Hu, The Classification and maximum determinants of the adjacency matrices of graphs with one cycle, J. Math. Study, 36 (2003), 102-104.

[10] M. Krivelevich and A. Nachmias, Colouring powers of cycles from random lists, European Journal of Combinatorics, 25 (2004), 961-968.

[11] D.Li and M.Liu, Hadwiger's conjecture for powers of cycles and their complements, European Journal of Combinatorics, 28 (2007), 1152-1155. 
